\title{
SUFFICIENT CONDITIONS FOR A REAL POLYNOMIAL TO BE A SUM OF SQUARES OF POLYNOMIALS
}

\author{
VAN DoAT DANG* AND ThI ThaO NGUYen ${ }^{\dagger}$
}

\begin{abstract}
In this paper, we establish new sufficient conditions for the polynomial $f$ to be SOS in terms of the Newton polyhedron of $f$ (Theorems 2.6 and 2.12). These new sufficient conditions include results which were proved earlier by Lasserre [13, Theorem 3], Fidalgo and Kovacec [6, Theorem 4.3], Ghasemi and Marshall [7, Theorems 2.1 and 2.3], and Ghasemi and Marshall [8, Theorem 2.3].
\end{abstract}

\section{Introduction}

The problem of representation of positive semidefinite polynomials (PSD) as sums of squares of polynomials (SOS) has been treated by many authors. Note that if $f$ is SOS then the degree of $f$ is even. In [10], Hilbert proved that $P_{2 d, n}=\Sigma_{2 d, n}$ if and only if $n \leq 2$ or $d=1$ or $(n, d)=(3,2)$, where $P_{2 d, n}$ and $\Sigma_{2 d, n}$ are the cone of PSD forms of degree $2 d$ in $n$ variables and the cone of SOS forms of degree $2 d$ in $n$ variables, respectively; see [2, Theorem 6.3.7]. The first explicit example for this phenomenon was given by Motzkin [18]; see [16, Proposition 1.2.2].

We denote by $\mathbf{N}$ the set of nonnegative integers $\{0,1,2, \ldots\}$. For $x=$ $\left(x_{1}, \ldots, x_{n}\right)$ and $\alpha=\left(\alpha_{1}, \ldots, \alpha_{n}\right) \in \mathbf{N}^{n}$, define $x^{\alpha}=x_{1}^{\alpha_{1}} \cdots x_{n}^{\alpha_{n}}$ and $|\alpha|=\alpha_{1}+\cdots+$ $\alpha_{n}$. Using these notations, every polynomial $f \in \mathbf{R}[x]$ can be writen as $f(x)=$ $\sum_{\alpha \in \mathbf{N}^{n}} f_{\alpha} x^{\alpha}$, where $f_{\alpha} \in \mathbf{R}$ and $f_{\alpha}=0$, except for finitely many $\alpha$. Assume now that $f$ is nonconstant and has even degree $2 d$. Let $\Omega:=\left\{\alpha \in \mathbf{N}^{n} \mid f_{\alpha} \neq 0\right\} \backslash$

2010 Mathematics Subject Classification. Primary 12D15; Secondary 14P99, 90C25.

Key words and phrases. Positive polynomial, sum of squares, Newton polyhedron, framework, mediated set, barycentric coordinates, optimization, geometric programming.

* This author was partially supported by Vietnam National Foundation for Science and Technology Development (NAFOSTED) grant number 101.04-2013.07.

$\dagger$ This author was partially supported by Vietnam National Foundation for Science and Technology Development (NAFOSTED) grant number 101.04-2014.23.

Received January 23, 2015; revised April 30, 2015. 
$\left\{0,2 d e_{1}, \ldots, 2 d e_{n}\right\}$, where $e_{1}=(1,0, \ldots, 0), \ldots, e_{n}=(0, \ldots, 0,1)$. Thus $f$ has the form

$$
f(x)=f_{0}+\sum_{\alpha \in \Omega} f_{\alpha} x^{\alpha}+\sum_{i=1}^{n} f_{2 d e_{i}} x_{i}^{2 d}
$$

Setting

$$
\begin{aligned}
\Delta & :=\left\{\alpha \in \Omega \mid f_{\alpha} x^{\alpha} \text { is not a sum of squares in } \mathbf{R}[x]\right\} \\
& =\left\{\alpha \in \Omega \mid f_{\alpha}<0 \text { or } \alpha_{i} \text { is odd for some } i \in\{1, \ldots, n\}\right\} .
\end{aligned}
$$

In [13, Theorem 3], Lasserre proved that if

$$
f_{0} \geq \sum_{\alpha \in \Delta}\left|f_{\alpha}\right| \quad \text { and } \quad f_{2 d e_{i}} \geq \sum_{\alpha \in \Delta}\left|f_{\alpha}\right| \frac{|\alpha|}{2 d}, \quad i=1, \ldots, n,
$$

then $f$ is SOS.

Later on, some various versions on sufficient conditions of this type have been given by Fidalgo and Kovacec [6, Theorem 4.3], and Ghasemi and Marshall [7, Theorems 2.1 and 2.3] where the left-hand sides of the inequalities are kept unchanged and the right-hand sides are replaced by quantities which also depend on the coefficients $f_{\alpha}, \alpha \in \Delta$. Indeed, in [7, Theorems 2.1], $f$ is SOS if

$$
f_{0} \geq \sum_{\alpha \in \Delta}\left|f_{\alpha}\right| \frac{2 d-|\alpha|}{2 d} \quad \text { and } \quad f_{2 d e_{i}} \geq \sum_{\alpha \in \Delta}\left|f_{\alpha}\right| \frac{\alpha_{i}}{2 d}, \quad i=1, \ldots, n
$$

and this result is an new version of Lasserre's one.

Recently, Ghasemi and Marshall [8, Theorem 2.3] have given another sufficient condition on additional variables satisfying constrains depending on the coefficients $f_{\alpha}, \alpha \in \Delta$, for the polynomial $f$ to be SOS. Precisely, a sufficient condition for a form $f$ of degree $2 d$ to be SOS is that there exist nonnegative real numbers $a_{\alpha, i}$ for $\alpha \in \Delta, i=1, \ldots, n$, such that

$$
(2 d)^{2 d} a_{\alpha}^{\alpha}=\left|f_{\alpha}\right|^{2 d} \alpha^{\alpha}, \quad \alpha \in \Delta, \quad \text { and } \quad f_{2 d e_{i}} \geq \sum_{\alpha \in \Delta} a_{\alpha, i}, \quad i=1, \ldots, n,
$$

where $a_{\alpha}^{\alpha}=a_{\alpha, 1}^{\alpha_{1}} \cdots a_{\alpha, n}^{\alpha_{n}}$ and $\alpha^{\alpha}=\alpha_{1}^{\alpha_{1}} \cdots \alpha_{n}^{\alpha_{n}}$ with the convention $0^{0}=1$. This result include improvements of the above results. However, if $f_{0}=f_{2 d e_{1}}=\cdots=$ $f_{2 d e_{n}}=0$, the conditions of $[8$, Theorem 2.3] imply that $\Delta=\emptyset$ and so $f$ is obviously SOS. Thus, this criterion is overly restrictive.

We recall here some notations about Newton polyhedra. Let $\operatorname{supp}(f):=$ $\left\{\alpha \in \mathbf{N}^{n} \mid f_{\alpha} \neq 0\right\}$. The Newton polyhedron $\Gamma(f)$ of $f$ is the convex hull of $\operatorname{supp}(f)$ in $\mathbf{R}^{n}$. Clearly, $\Gamma(f)$ is a compact convex polyhedron of dimension at most $n$. A supporting hyperplane of $\Gamma(f)$ is a hyperplane minimizing the value of some linear function on $\Gamma(f)$. The faces of the boundary of the Newton polyhedron $\Gamma(f)$ are the intersection of $\Gamma(f)$ with its supporting hyperplanes. 
They are compact convex polyhedra of dimension at most $n-1$. The vertices are faces of dimension 0 (i.e., points).

In this paper, we establish new sufficient conditions for the polynomial $f$ to be SOS in terms of the Newton polyhedron $\Gamma(f)$ of $f$ (Theorems 2.6 and 2.12). Precisely, we replace the values $f_{0}, f_{2 d e_{1}}, \ldots, f_{2 d e_{n}}$ in the left sides of the inequalities above by some values $f_{u}, u \in \mathscr{U}$, where $\mathscr{U}$ is some set satisfying $V(f) \subset \mathscr{U} \subset$ $\Gamma(f) \cap(2 \mathbf{Z})^{n}$. Note that our new sufficient conditions include all the results mentioned above.

The paper is organized as follows. Section 2 presents the new sufficient conditions for a polynomial to be a sum of squares of polynomials (Theorem 2.6 and 2.12). In Section 3, we prove these results. As a consequence of Theorem 2.12, a lower bound of $f$ is established in Section 4 .

\section{Statement of the results}

Suppose $f\left(x_{1}, \ldots, x_{n}\right)$ is a polynomial of degree $2 d$. Let

$$
\bar{f}\left(x_{0}, x_{1}, \ldots, x_{n}\right):=x_{0}^{2 d} f\left(\frac{x_{1}}{x_{0}}, \ldots, \frac{x_{n}}{x_{0}}\right) .
$$

Then $\bar{f}$ is a form of degree $2 d$ called the homogenization of $f$. We have the following well-known result.

Proposition 2.1 ([16, Proposition 1.2.4]). Let $f$ be a polynomial of degree 2d. Then $f$ is PSD iff $\bar{f}$ is PSD; and $f$ is SOS iff $\bar{f}$ is SOS.

We now assume $f$ is a form, and $\Gamma(f)$ is the Newton polyhedron of $f$. Let

- $\mathscr{C}:=\Gamma(f) \cap \mathbf{Z}^{n}$ denote the set of all integer points in $\Gamma(f)$,

- $\mathscr{A}:=\left\{\frac{1}{2}(s+t) \mid s, t \in \Gamma(f) \cap(2 \mathbf{Z})^{n}\right\}$ denote the set of integer points from $\Gamma(f)$ which are averages of even integer points from $\Gamma(f)$, and

- $\mathscr{V}:=\mathscr{A} \backslash\left\{\frac{1}{2}(s+t) \mid s \neq t, s, t \in \Gamma(f) \cap(2 \mathbf{Z})^{n}\right\}$ denote the set of even integer points from $\Gamma(f)$ which are not averages of distinct even integer points from $\Gamma(f)$.

From now on, $V:=V(f)$ denotes the set of vertices of the Newton polyhedron of $f$. It follows from [9, Theorem 3.1] that if $f$ is PSD, the set $V$ is contained in $(2 \mathbf{Z})^{n}$, and so $V \subset \mathscr{V} \subset \mathscr{C} \cap(2 \mathbf{Z})^{n}$. We recall some results, which give some necessary conditions for a polynomial to be SOS.

Proposition 2.2 ([4, Proposition 3.7]). If $f$ is SOS, then

(i) for every $\alpha \in \operatorname{supp}(f)$, we have $\alpha \in \mathscr{A}$, and

(ii) for every $u \in \mathscr{V}, f_{u} \geq 0$.

We recall here some notations, due to Reznick [23]. We use the term framework to denote a set $\mathscr{U}=\left\{u^{1}, \ldots, u^{m}\right\}$ in which $u^{i}=\left(u_{1}^{i}, \ldots, u_{n}^{i}\right) \in(2 \mathbf{Z})^{n}$ with $u_{j}^{i} \geq 0$ and $\sum_{j=1}^{n} u_{j}^{i}=2 d$ for all $i$ and some $d$. A trellis is a framework in 
which $u^{1}, \ldots, u^{m}$ comprise the vertices of a simplex. Note that if $\mathscr{U}$ is a trellis, then $\mathscr{U}$ is a linearly independent set.

Definition 2.3 ([23, Definition 2.1]). Let $\mathscr{U}$ be a framework. A finite set $\mathscr{L} \subset \mathbf{Z}^{n}$ is called $\mathscr{U}$-mediated if $\mathscr{L}$ contains $\mathscr{U}$, and every $v \in \mathscr{L} \backslash \mathscr{U}$ is an average of two distinct even points in $\mathscr{L}$.

We now assume that $V \subset \mathscr{U} \subset \mathscr{V}$. Then, $\mathscr{U}$ is a framework. By [23, Theorem 2.2], we get the following.

THEOREM 2.4 ([23]). There is a $\mathscr{U}$-mediated set $\mathscr{U}^{*}$ satisfying $\mathscr{A}(\mathscr{U}):=$ $\left\{\frac{1}{2}(s+t) \mid s, t \in \mathscr{U}\right\} \subset \mathscr{U}^{*} \subset \mathscr{C}$ and $\mathscr{U}^{*}$ contains every $\mathscr{U}$-mediated set.

Suppose that $V=\left\{u^{1}, \ldots, u^{m}\right\}$ is a framework. If $\alpha \in \mathbf{Z}^{n}$ and $\alpha=\lambda_{1} u^{1}+$ $\cdots+\lambda_{m} u^{m}$ with $\lambda_{i} \geq 0$ and $\lambda_{1}+\cdots+\lambda_{m}=1$, then $\alpha \in \mathscr{C}$; conversely, if $\alpha \in \mathscr{C}$ then at least one such $\lambda=\left(\lambda_{1}, \ldots, \lambda_{m}\right)$ exists. Let

$$
\Lambda(\alpha)=\left\{\lambda=\left(\lambda_{1}, \ldots, \lambda_{m}\right) \mid \lambda_{i} \geq 0, \lambda_{1}+\cdots+\lambda_{m}=1, \text { and } \alpha=\lambda_{1} u^{1}+\cdots+\lambda_{m} u^{m}\right\} .
$$

If $V$ is a trellis, then only one such $\lambda=\left(\lambda_{1}, \ldots, \lambda_{m}\right)$ exists, and $\left(\lambda_{1}, \ldots, \lambda_{m}\right)$ are called the barycentric coordinates of $\alpha$ with respect to $V$.

Theorem 2.5 ([23, Theorem 3.3]). Suppose $V=\left\{u^{1}, \ldots, u^{m}\right\}$ is a framework, $\alpha \in \mathscr{C}$ and $\lambda \in \Lambda(\alpha)$. Then, if the form $f(V, \lambda, \alpha)(x):=\lambda_{1} x^{u^{1}}+\cdots+\lambda_{m} x^{u^{m}}-x^{\alpha}$ is $\operatorname{SOS}$ then $\alpha \in V^{*}$.

The following is our new result, which gives a sufficient condition on the coefficients for a polynomial to be sum of squares.

THEOREM 2.6. Let $f$ be a form in $n$ real variables with $V \subset(2 \mathbf{Z})^{n}$. Suppose that $V \subset \mathscr{U} \subset \mathscr{V}$ such that

(i) $\alpha \in \mathscr{U}^{*}$ for all $\alpha \in \Delta$,

(ii) $\min _{u \in \mathscr{U}} f_{u} \geq \sum_{\alpha \in \Delta}\left|f_{\alpha}\right|$.

Then $f$ is SOS. Here, by convention, we set $\sum_{\alpha \in \Delta}\left|f_{\alpha}\right|:=0$ if $\Delta=\emptyset$.

COROllary 2.7. Let $f$ be a form in $n$ real variables with $V \subset(2 \mathbf{Z})^{n}$. Suppose

(i) $\alpha \in \mathscr{A}$ for all $\alpha \in \Delta$,

(ii) $\min _{u \in \mathscr{V}} f_{u} \geq \sum_{\alpha \in \Delta}\left|f_{\alpha}\right|$.

Then $f$ is SOS.

COROllary 2.8. Let $f$ be a form in 3 real variables with $V \subset(2 \mathbf{Z})^{3}$. Then, $f$ is SOS if

$$
\min _{u \in \mathscr{V}} f_{u} \geq \sum_{\alpha \in \Delta}\left|f_{\alpha}\right|
$$


Remark 2.9. Condition (ii) of Theorem 2.6 together with $f_{u}=0$ for some $u \in \mathscr{U}$ implies that $\Delta=\emptyset$ and $f_{u} \geq 0$ for all $u \in \mathscr{U}$; in this case, $f$ is obviously SOS. Thus, an alternative sufficient condition is given by Theorem 2.6 for $V \subset \mathscr{U} \subset \operatorname{supp}(f) \cap \mathscr{V}$.

Next, we shall give another sufficient condition for a polynomial to be SOS. We recall the following result, due to Reznick.

THEOREm 2.10 ([23, Corollary 4.9]). Suppose $V=\left\{u^{1}, \ldots, u^{m}\right\}$ is a trellis, and $\alpha \in \mathscr{C}$. Then the form $f(V, \alpha)(x):=\lambda_{1} x^{u^{1}}+\cdots+\lambda_{m} x^{u^{m}}-x^{\alpha}$ is SOS if and only if $\alpha \in V^{*}$. Here $\left(\lambda_{1}, \ldots, \lambda_{m}\right)$ are the barycentric coordinates of $\alpha$ with respect to $V$.

We will make use Theorem 2.10 to prove the following.

Corollary 2.11. Suppose that $V=\left\{u^{1}, \ldots, u^{m}\right\}$ is a trellis and $\alpha \in V^{*}$. Then, for a form $E(x)=b_{1} x^{u^{1}}+\cdots+b_{m} x^{u^{m}}-c x^{\alpha}$ such that $b_{i} \geq 0$ for $i=$ $1, \ldots, m$, and $c \geq 0$ if all $\alpha_{i}$ are even, the following are equivalent:

(i) $E \geq 0$.

(ii) $|c| \lambda_{1}^{\lambda_{1}} \cdots \lambda_{m}^{\lambda_{m}} \leq b_{1}^{\lambda_{1}} \cdots b_{m}^{\lambda_{m}}$ (with the convention $0^{0}=1$ ).

(iii) $E$ is $S O S$.

Here $\left(\lambda_{1}, \ldots, \lambda_{m}\right)$ are the barycentric coordinates of $\alpha$ with respect to $V$.

Now, we present our other result, which is an improved version of $[8$, Theorem 2.3]. The proof of this result is by using Corollary 2.11.

THEOREM 2.12. Let $f$ be a form, and $V=\left\{u^{1}, \ldots, u^{m}\right\}$ be the set of vertices of $\Gamma(f)$. Suppose that $V$ is a trellis, and $\alpha \in V^{*}$ for all $\alpha \in \Delta$. A sufficient condition for $f$ to be SOS is that there exist nonnegative real numbers $a_{\alpha, i}$ for $\alpha \in \Delta, i=1, \ldots, m$, such that

(i) $a_{\alpha}^{\lambda_{\alpha}}=\left|f_{\alpha}\right| \lambda_{\alpha}^{\lambda_{\alpha}}, \alpha \in \Delta$, and

(ii) $f_{u^{i}} \geq \sum_{\alpha \in \Delta} a_{\alpha, i}, i=1, \ldots, m$.

Here $a_{\alpha}=\left(a_{\alpha, 1}, \ldots, a_{\alpha, m}\right), \lambda_{\alpha}=\left(\lambda_{\alpha, 1}, \ldots, \lambda_{\alpha, m}\right)$ are the barycentric coordinates of $\alpha$ with respect to $V, a_{\alpha}^{\lambda_{\alpha}}=a_{\alpha, 1}^{\lambda_{\alpha, 1}} \cdots a_{\alpha, m}^{\lambda_{\alpha, m}}$, and $\lambda_{\alpha}^{\lambda_{\alpha}}=\lambda_{\alpha, 1}^{\lambda_{\alpha, 1}} \cdots \lambda_{\alpha, m}^{\lambda_{\alpha, m}}$.

We mention some corollaries of Theorem 2.12. Corollary 2.13 generalizes known earlier results, due to Lasserre [13, Theorem 3] and Ghasemi and Marshall [7, Theorem 2.1]. Corollary 2.14 includes results which were proved earlier by Fidalgo and Kovacec [6, Theorem 4.3] and Ghasemi and Marshall [7, Theorem 2.3]. Corollary 2.15 is a generalization of the result of Ghasemi and Marshall [8, Corollary 2.7]. Each corollary will be proved by applying Theorem 2.12 for a particular choice of the lifted variables $a_{\alpha, i}$. 
Corollary 2.13. Suppose $f$ is a form such that $V=\left\{u^{1}, \ldots, u^{m}\right\}$ is a trellis, and $\alpha \in V^{*}$ for all $\alpha \in \Delta$. Then if

$$
f_{u^{i}} \geq \sum_{\alpha \in \Delta}\left|f_{\alpha}\right| \lambda_{\alpha, i}, \quad i=1, \ldots, m
$$

then $f$ is SOS.

Corollary 2.14. Suppose $f$ is a form such that $V=\left\{u^{1}, \ldots, u^{m}\right\}$ is a trellis, and $\alpha \in V^{*}$ for all $\alpha \in \Delta$. Then $f$ is SOS if

$$
f_{u^{i}} \geq \sum_{\alpha \in \Delta}\left|f_{\alpha}\right| \lambda_{\alpha}^{\lambda_{\alpha}}, \quad i=1, \ldots, m
$$

Corollary 2.15. Suppose $f$ is a form such that $V=\left\{u^{1}, \ldots, u^{m}\right\}$ is a trellis, and $\alpha \in V^{*}$ for all $\alpha \in \Delta$. Then $f$ is SOS if

$$
\sum_{\alpha \in \Delta} \frac{\left|f_{\alpha}\right| \lambda_{\alpha}^{\lambda_{\alpha}}}{\prod_{i=1}^{m} f_{u^{i}}^{\lambda_{\alpha, i}}} \leq 1
$$

Notice that, by a similar argument as in [8, Remark 2.8], Corollary 2.15 is an improved version of Corollary 2.14.

Corollary 2.16. Suppose $f$ is a form such that $V=\left\{u^{1}, \ldots, u^{m}\right\}$ is a trellis, and $\alpha \in V^{*}$ for all $\alpha \in \Delta$. Then $f$ is SOS if

$$
f_{u^{i}} \geq \sum_{\alpha \in \Delta, \lambda_{\alpha, i} \neq 0} k \lambda_{\alpha, i}\left(\frac{\left|f_{\alpha}\right|}{k}\right)^{1 / \lambda_{\alpha, i} n_{\alpha}}, \quad i=1, \ldots, m,
$$

where $n_{\alpha}:=\left|\left\{i \mid \lambda_{\alpha, i} \neq 0\right\}\right|$, and $k$ is a positive real number.

\section{Proof of the results}

Let $\mathbf{R}[x]$ be the ring of real polynomials in the variables $x=\left(x_{1}, \ldots, x_{n}\right)$, and $\mathbf{R}[x]_{2 d}$ be the vector space of real polynomials of degree at most $2 d$, with canonical basis $\left(x^{\alpha}\right)=\left\{x^{\alpha}\left|\alpha \in \mathbf{N}^{n},\right| \alpha \mid \leq 2 d\right\}$. Given a sequence $y=\left(y_{\alpha}\right) \subset \mathbf{R}$ indexed by the canonical basis $\left(x^{\alpha}\right)$, let $L_{y}: \mathbf{R}[x]_{2 d} \rightarrow \mathbf{R}$ be the linear mapping

$$
f=\sum_{\alpha} f_{\alpha} x^{\alpha} \mapsto L_{y}(f)=\sum_{\alpha} f_{\alpha} y_{\alpha}, \quad f \in \mathbf{R}[x]_{2 d},
$$

and let $M_{d}(y)$ be the moment matrix with rows and columns indexed by $\left(x^{\alpha}\right)$, and defined by

$$
M_{d}(y)(\alpha, \beta):=L_{y}\left(x^{\alpha+\beta}\right)=y_{\alpha+\beta}, \quad \alpha, \beta \in \mathbf{N}^{n}:|\alpha|,|\beta| \leq d .
$$

Let the notation $M_{d}(y) \succeq 0$ stand for $M_{d}(y)$ is positive semidefinite. 
Remark 3.1. It is clear that $M_{d}(y) \succeq 0$ iff $L_{y}\left(f^{2}\right) \geq 0$ for all $f \in \mathbf{R}[x]_{d}$. Moreover, $f$ is SOS iff $L_{y}(f) \geq 0$ for all $y$ such that $M_{d}(y) \succeq 0$ (this is a corollary of $[14$, Theorem 3.1]).

Lemma 3.2. Let $\mathscr{U}$ be a framework and $\mathscr{L}$ be a $\mathscr{U}$-mediated set. Suppose $y=\left(y_{\alpha}\right) \subset \mathbf{R}$ such that $M_{d}(y) \succeq 0$. Then

$$
\left|L_{y}\left(x^{\alpha}\right)\right| \leq \max _{u \in \mathscr{U}} L_{y}\left(x^{u}\right) \quad \text { for all } \alpha \in \mathscr{L} .
$$

Proof. First of all, we will show that if $\alpha \in \mathscr{L} \backslash \mathscr{U}$, there exists $k \geq 1$ and a sequence

$$
\alpha_{i-1}=\frac{1}{2}\left(\alpha_{i}+\beta_{i}\right), \quad \alpha_{i} \neq \beta_{i}, \quad \alpha_{i}, \beta_{i} \in \mathscr{L} \cap(2 \mathbf{Z})^{n}, \quad i=1, \ldots, k,
$$

such that $\alpha_{0}=\alpha$ and $\alpha_{k} \in \mathscr{U}$. In fact, let

$$
\begin{gathered}
X=\left\{\alpha^{\prime} \mid \text { there exists } k \geq 1 \text { and a sequence } \alpha_{i-1}=\frac{1}{2}\left(\alpha_{i}+\beta_{i}\right), \alpha_{i} \neq \beta_{i},\right. \\
\left.\alpha_{i}, \beta_{i} \in \mathscr{L} \cap(2 \mathbf{Z})^{n}, i=1, \ldots, k, \text { such that } \alpha_{0}=\alpha \text { and } \alpha_{k}=\alpha^{\prime}\right\} .
\end{gathered}
$$

Since $X$ is contained in $\mathscr{L}$, the set $X$ is finite, and so the convex hull of $X$ has vertices which belong to $\mathscr{U}$.

Now, setting $\tau:=\max _{\alpha \in \mathscr{L}}\left|L_{y}\left(x^{\alpha}\right)\right|$. Then there exists some $\gamma \in \mathscr{L}$ such that $\tau=\left|L_{y}\left(x^{\gamma}\right)\right| . \quad$ If $\gamma \in \mathscr{U}$, then

$$
\left|L_{y}\left(x^{\alpha}\right)\right| \leq\left|L_{y}\left(x^{\gamma}\right)\right|=\max _{u \in \mathscr{U}}\left|L_{y}\left(x^{u}\right)\right| \text { for all } \alpha \in \mathscr{L} .
$$

Otherwise, by the above claim, there exists some $k \geq 1$ such that

$$
\begin{aligned}
\gamma= & \frac{1}{2}\left(\gamma_{1}+\beta_{1}\right), \quad \gamma_{1} \neq \beta_{1} \in \mathscr{L} \cap(2 \mathbf{Z})^{n}, \\
\gamma_{1}= & \frac{1}{2}\left(\gamma_{2}+\beta_{2}\right), \quad \gamma_{2} \neq \beta_{2} \in \mathscr{L} \cap(2 \mathbf{Z})^{n}, \\
& \ldots \ldots \ldots \ldots \\
\gamma_{k-1}= & \frac{1}{2}\left(\gamma_{k}+\beta_{k}\right), \quad \gamma_{k} \neq \beta_{k} \in \mathscr{L} \cap(2 \mathbf{Z})^{n},
\end{aligned}
$$

where $\gamma_{k} \in \mathscr{U}$. Since $M_{d}(y) \succeq 0$, we have

$$
\tau=\left|L_{y}\left(x^{\gamma}\right)\right|=\left|L_{y}\left(x^{(1 / 2)\left(\gamma_{1}+\beta_{1}\right)}\right)\right| \leq \sqrt{L_{y}\left(x^{\gamma_{1}}\right) L_{y}\left(x^{\beta_{1}}\right)} \leq \sqrt{L_{y}\left(x^{\gamma_{1}}\right) \tau} .
$$

Hence $\tau \leq L_{y}\left(x^{\gamma_{1}}\right)$. By repeating the above arguments, after finitely many steps, we deduce

$$
\tau \leq L_{y}\left(x^{\gamma_{k}}\right) \leq \max _{u \in \mathscr{U}} L_{y}\left(x^{u}\right)
$$

This complete the proof of the lemma. 
Proof of Theorem 2.6. By Remark 3.1, it is sufficient to show that $L_{y}(f) \geq 0$ for any $y$ such that $M_{d}(y) \succeq 0$.

So let $y$ be such that $M_{d}(y) \succeq 0$. Let $\tau:=\max \left\{L_{y}\left(x^{u}\right) \mid u \in \mathscr{U}\right\}$. Then, by Lemma 3.2, we have

$$
\left|L_{y}\left(x^{\alpha}\right)\right| \leq \tau \text { for all } \alpha \in \mathscr{U}^{*}
$$

This together with Conditions (i)-(ii) implies that

$$
\begin{aligned}
L_{y}(f) & =\sum_{u \in \mathscr{U}} f_{u} L_{y}\left(x^{u}\right)+\sum_{\alpha \in \Delta} f_{\alpha} L_{y}\left(x^{\alpha}\right)+\sum_{\alpha \notin(\mathscr{U} \cup \Delta)} f_{\alpha} L_{y}\left(x^{\alpha}\right) \\
& \geq\left(\min _{u \in \mathscr{U}} f_{u}-\sum_{\alpha \in \Delta}\left|f_{\alpha}\right|\right) \tau \geq 0 .
\end{aligned}
$$

Proof of Corollary 2.7. We first prove the following.

Claim 3.3. We have $\mathscr{V}^{*}=\mathscr{A}$.

Proof. We first prove that $\mathscr{A} \subset \mathscr{V}^{*}$. Indeed, for every $\alpha \in \mathscr{A} \backslash \mathscr{V}$, we have $\alpha=\frac{1}{2}(s+t)$ for some $s \neq t, s, t \in \Gamma(f) \cap(2 \mathbf{Z})^{n} \subset \mathscr{A} \cap(2 \mathbf{Z})^{n}$, and hence $\alpha$ is an average of two distinct even points in $\mathscr{A}$. Thus $\mathscr{A}$ is a $\mathscr{V}$-mediated set, and so $\mathscr{A} \subset \mathscr{V}^{*}$.

On the other hand, let $\mathscr{C}^{\prime}:=\mathscr{C} \backslash \mathscr{A}$, we will show that $\mathscr{C}^{\prime} \cap \mathscr{V}^{*}=\emptyset$. Indeed, by contradiction, assume that there exists some $\alpha \in \mathscr{C}^{\prime} \cap \mathscr{V}^{*}$. Then, we have

- $\alpha \in \mathscr{C}^{\prime}$, and so $\alpha \notin \mathscr{A}$;

- $\alpha \in \mathscr{V}^{*}$; and hence $\alpha=\frac{1}{2}(s+t)$ for some $s, t \in \mathscr{V}^{*} \cap(2 \mathbf{Z})^{n} \subset \mathscr{C} \cap(2 \mathbf{Z})^{n}$, by Theorem 2.4; and so $\alpha \in \mathscr{A}$.

The contradiction shows that $\mathscr{C}^{\prime} \cap \mathscr{V}^{*}=\emptyset$, and hence $\mathscr{V}^{*} \subset \mathscr{A}$.

Therefore, we have $\mathscr{V}^{*}=\mathscr{A}$.

Now, the corollary follows imediately from Theorem 2.6 and Claim 3.3.

Proof of Corollary 2.8. Note that $\mathscr{V}$ is also framework in $\mathbf{Z}^{3}$. Then it is shown in [22] that $\mathscr{A}=\mathscr{C}$. This, together with Claim 3.3, shows that $\mathscr{V}^{*}=\mathscr{C}$. Thus, Condition (i) of Theorem 2.6 is obviously true, and $f$ is SOS.

Proof of Corollary 2.11. (iii) $\Rightarrow$ (i) is trivial, so it suffices to show that (i) $\Rightarrow$ (ii) and (ii) $\Rightarrow$ (iii).

First of all, notice that:

- If $c=0$, the result is trivially true.

- If $c<0$, by the assumption, there exists some $\alpha_{i} \notin 2 Z$. Then making a change of the variables $x_{i}=-y_{i}$, and $x_{j}=y_{j}$ for $j \neq i, c$ is replaced by $-c$. In this way, we can assume that $c>0$. 
- If $\lambda_{m}=0$, let

$$
\tilde{E}(x)=b_{1} x^{u^{1}}+\cdots+b_{m-1} x^{u^{m-1}}-c x^{\alpha} .
$$

Claim 3.4. The following are equivalent.

(a) $E \geq 0$.

(b) $\tilde{E} \geq 0$.

Proof. $\quad(\mathrm{a}) \Rightarrow(\mathrm{b})$. Suppose $E(x) \geq 0$ for all $x \in \mathbf{R}^{n}$. Since $\mathscr{U}=\left\{u^{1}, \ldots, u^{m}\right\}$ is a trellis, $\left\{u^{1}, \ldots, u^{m}\right\}$ is linearly independent. Without the loss of genrerality, we can assume that

$$
\operatorname{det}\left[\begin{array}{ccc}
u_{1}^{1} & \cdots & u_{m}^{1} \\
\cdots & \cdots & \cdots \\
u_{1}^{m} & \cdots & u_{m}^{m}
\end{array}\right] \neq 0,
$$

where $u^{i}=\left(u_{1}^{i}, \ldots, u_{n}^{i}\right), i=1, \ldots, m$. We will show that there exist $c_{1}, \ldots, c_{m} \in$ $\mathbf{R}_{>0}$ such that

$$
\left\{\begin{array}{l}
c_{1}^{u_{1}^{1}} \cdots c_{m}^{u_{m}^{1}}=1 \\
\cdots \cdots \cdots \cdots \cdots \\
c_{1}^{u_{1}^{m-1}} \cdots c_{m}^{u_{m}^{m-1}}=1 \\
c_{1}^{u_{1}^{m}} \cdots c_{m}^{u_{m}^{m}}=\varepsilon
\end{array}\right.
$$

for every $\varepsilon \in \mathbf{R}_{>0}$. In fact, it is clear that the system of linear equations

$$
\left\{\begin{array}{l}
u_{1}^{1} \theta_{1}+\cdots+u_{m}^{1} \theta_{m}=0 \\
\cdots \cdots \cdots+\cdots \\
u_{1}^{m-1} \theta_{1}+\cdots+u_{m}^{m-1} \theta_{m}=0 \\
u_{1}^{m} \theta_{1}+\cdots+u_{m}^{m} \theta_{m}=\ln \varepsilon
\end{array}\right.
$$

has one only root $\left(\theta_{1}, \ldots, \theta_{m}\right) \in \mathbf{R}^{m}$. Let $c_{i}:=e^{\theta_{i}}, i=1, \ldots, m$, so the claim is proved. Make a change of variables

$$
\left\{\begin{array}{l}
x_{i}=c_{i} y_{i}, \quad i=1, \ldots, m \\
x_{j}=y_{j}, \quad j=m+1, \ldots, n .
\end{array}\right.
$$

Then

$$
\begin{aligned}
E_{\varepsilon}(y):= & E(x)=b_{1} x^{u^{1}}+\cdots+b_{m} x^{u^{m}}-c\left(x^{u^{1}}\right)^{\lambda_{1}} \cdots\left(x^{u^{m-1}}\right)^{\lambda_{m-1}} \\
= & b_{1}\left(c_{1}^{u_{1}^{1}} \cdots c_{m}^{u_{m}^{1}}\right) y^{u^{1}}+\cdots+b_{m}\left(c_{1}^{u_{1}^{m}} \cdots c_{m}^{u_{m}^{m}}\right) y^{u^{m}} \\
& -c\left[\left(c_{1}^{u_{1}^{1}} \cdots c_{m}^{u_{m}^{1}}\right) y^{u^{1}}\right]^{\lambda_{1}} \cdots\left[\left(c_{1}^{u_{1}^{m-1}} \cdots c_{m}^{u_{m}^{m-1}}\right) y^{u^{m-1}}\right]^{\lambda_{m-1}} \\
= & b_{1} y^{u^{1}}+\cdots+b_{m-1} y^{u^{m-1}}+\varepsilon b_{m} y^{u^{m}}-c y^{\alpha} .
\end{aligned}
$$


Since $E_{\varepsilon}(y) \geq 0$ for all $y \in \mathbf{R}^{n}$, we have $\tilde{E}(y)=\lim _{\varepsilon \rightarrow 0} E_{\varepsilon}(y) \geq 0$ for all $y \in \mathbf{R}^{n}$. This proves (b).

(b) $\Rightarrow(\mathrm{a})$. It is clear.

Claim 3.5. Let $\tilde{\mathscr{U}}=\left\{u^{1}, \ldots, u^{m-1}\right\}$. Then $\tilde{\mathscr{U}}$ is a trellis and $\alpha \in \tilde{\mathscr{U}}^{*}$.

Proof. (a) Since $\mathscr{U}$ is a trellis, $\left\{u^{1}, \ldots, u^{m}\right\}$ comprise the vertices of a simplex. Thus, $\left\{u^{1}, \ldots, u^{m-1}\right\}$ also comprise the vertices of a simplex, and so $\tilde{\mathscr{U}}$ is a trellis.

(b) Let $\mathscr{L}=\mathscr{U}^{*} \cap \operatorname{conv}(\tilde{\mathscr{U}})$. Note that $\tilde{\mathscr{U}} \subset \mathscr{L}$. We will show that $\mathscr{L}$ is a $\tilde{\mathscr{U}}$-mediated set. In fact, for every $\beta \in \mathscr{L} \backslash \tilde{\mathscr{U}}$, we have $\beta \in \mathscr{U}^{*} \backslash \mathscr{U}$; and hence

$$
\beta=\frac{1}{2}(s+t) \text { for some } s \neq t \in \mathscr{U}^{*} \cap(2 \mathbf{Z})^{n} \text {. }
$$

Let $\lambda(s)=\left(\lambda_{1}(s), \ldots, \lambda_{m}(s)\right)$, and $\lambda(t)=\left(\lambda_{1}(t), \ldots, \lambda_{m}(t)\right)$ be the barycentric coordinates of $s$ and $t$ respect to $\mathscr{U}$. Then

$$
\lambda(\beta)=\left(\frac{\lambda_{1}(s)+\lambda_{1}(t)}{2}, \ldots, \frac{\lambda_{m}(s)+\lambda_{m}(t)}{2}\right)
$$

are the barycentric coordinates of $\beta$ respect to $\mathscr{U}$. Since $\beta \in \operatorname{conv}(\tilde{\mathscr{U}})$, we have $\lambda_{m}(s)+\lambda_{m}(t)=0$. This implies that

$$
\lambda_{m}(s)=\lambda_{m}(t)=0,
$$

since $\lambda_{m}(s), \lambda_{m}(t) \geq 0 ;$ and so $s, t \in \operatorname{conv}(\tilde{\mathscr{U}})$. Therefore, $s, t \in \mathscr{L} \cap(2 \mathbf{Z})^{n}$; and hence $\mathscr{L}=\mathscr{U}^{*} \cap \operatorname{conv}(\tilde{\mathscr{U}})$ is a $\tilde{\mathscr{U}}$-mediated set. In particular, $\alpha \in \mathscr{U}^{*} \cap \operatorname{conv}(\tilde{\mathscr{U}})$ $\subset \tilde{\mathscr{U}}^{*}$.

By Claim 3.4 and 3.5, it is sufficient to consider the case all $\lambda_{i}$ are non-zero.

- If $b_{m}=0$, then (ii) fails, since $c, \lambda_{1}, \ldots, \lambda_{m} \in \mathbf{R}_{>0}$. Taking

$$
x:=\left(c_{1}, \ldots, c_{m}, 1, \ldots, 1\right),
$$

where

$$
\left\{\begin{array}{l}
c_{1}^{u_{1}^{1}} \cdots c_{m}^{u_{m}^{1}}=1 \\
\cdots \cdots \cdots \cdots \cdots \\
c_{1}^{u_{1}^{m-1}} \cdots c_{m}^{u_{m}^{m-1}}=1 \\
c_{1}^{u_{1}^{m}} \cdots c_{m}^{u_{m}^{m}}=k
\end{array}\right.
$$

for every $k \in \mathbf{R}_{>0}$. Then

$$
\begin{aligned}
E(x)= & b_{1} x^{u^{1}}+\cdots+b_{m-1} x^{u^{m-1}}-c\left(x^{u^{1}}\right)^{\lambda_{1}} \cdots\left(x^{u^{m}}\right)^{\lambda_{m}} \\
= & b_{1}\left(c_{1}^{u_{1}^{1}} \cdots c_{m}^{u_{m}^{1}}\right)+\cdots+b_{m-1}\left(c_{1}^{u_{1}^{m-1}} \cdots c_{m}^{u_{m}^{m-1}}\right) \\
& -c\left[\left(c_{1}^{u_{1}^{1}} \cdots c_{m}^{u_{m}^{1}}\right)\right]^{\lambda_{1}} \cdots\left[\left(c_{1}^{u_{1}^{m}} \cdots c_{m}^{u_{m}^{m}}\right)\right]^{\lambda_{m}} \\
= & b_{1}+\cdots+b_{m-1}-c k^{\lambda_{m}} .
\end{aligned}
$$


So $E(x)<0$ for $k$ sufficiently large. This implies that (i) also fails. Thus the claimed implication are trivially true in this case.

Now, we can assume that $b_{1}, \ldots, b_{m}, c, \lambda_{1}, \ldots, \lambda_{m} \in \mathbf{R}_{>0}$. By the same arguments as above, there exist positive real numbers $c_{1}, \ldots, c_{m}$ such that

$$
\left\{\begin{array}{l}
c_{1}^{u_{1}^{1}} \cdots c_{m}^{u_{m}^{1}}=\frac{\lambda_{1}}{b_{1}} \\
\cdots \cdots \cdots \cdots \cdots \\
c_{1}^{u_{1}^{m}} \cdots c_{m}^{u_{m}^{m}}=\frac{\lambda_{m}}{b_{m}} .
\end{array}\right.
$$

(i) $\Rightarrow$ (ii). Assume $E(x) \geq 0$ for all $x \in \mathbf{R}^{n}$. Taking

$$
x:=\left(c_{1}, \ldots, c_{m}, 1, \ldots, 1\right) .
$$

Then, we have

$$
\begin{aligned}
E(x) & =b_{1} x^{u^{1}}+\cdots+b_{m} x^{u^{m}}-c\left(x^{u^{1}}\right)^{\lambda_{1}} \cdots\left(x^{u^{m}}\right)^{\lambda_{m}} \\
& =b_{1}\left(c_{1}^{u_{1}^{1}} \cdots c_{m}^{u_{m}^{1}}\right)+\cdots+b_{m}\left(c_{1}^{u_{1}^{m}} \cdots c_{m}^{u_{m}^{m}}\right)-c\left[c_{1}^{u_{1}^{1}} \cdots c_{m}^{u_{m}^{1}}\right]^{\lambda_{1}} \cdots\left[c_{1}^{u_{1}^{m}} \cdots c_{m}^{u_{m}^{m}}\right]^{\lambda_{m}} \\
& =\lambda_{1}+\cdots+\lambda_{m}-c\left(\frac{\lambda_{1}}{b_{1}}\right)^{\lambda_{1}} \cdots\left(\frac{\lambda_{m}}{b_{m}}\right)^{\lambda_{m}} \\
& =1-c\left(\frac{\lambda_{1}}{b_{1}}\right)^{\lambda_{1}} \cdots\left(\frac{\lambda_{m}}{b_{m}}\right)^{\lambda_{m}} \\
& =1-c \frac{\lambda^{\lambda}}{b^{\lambda}} \geq 0 .
\end{aligned}
$$

This proves (ii).

(ii) $\Rightarrow$ (iii). Make a change of variables

$$
\left\{\begin{array}{l}
x_{i}=c_{i} y_{i}, \quad i=1, \ldots, m \\
x_{j}=y_{j}, \quad j=m+1, \ldots, n
\end{array}\right.
$$

Let $\tilde{c}:=c\left(\frac{\lambda_{1}}{b_{1}}\right)^{\lambda_{1}} \cdots\left(\frac{\lambda_{m}}{b_{m}}\right)^{\lambda_{m}}$. By (ii), we have $\tilde{c} \leq 1$. Then

$$
\begin{aligned}
E(x)= & b_{1}\left(c_{1}^{u_{1}^{1}} \cdots c_{m}^{u_{m}^{1}}\right) y^{u^{1}}+\cdots+b_{m}\left(c_{1}^{u_{1}^{m}} \cdots c_{m}^{u_{m}^{m}}\right) y^{u^{u^{m}}} \\
& -c\left[\left(c_{1}^{u_{1}^{1}} \cdots c_{m}^{u_{m}^{1}}\right) y^{u^{1}}\right]^{\lambda_{1}} \cdots\left[\left(c_{1}^{u_{1}^{m}} \cdots c_{m}^{u_{m}^{m}}\right) y^{u^{m}}\right]^{\lambda_{m}} \\
= & \lambda_{1} y^{u^{1}}+\cdots+\lambda_{m} y^{u^{m}}-c\left(\frac{\lambda_{1}}{b_{1}}\right)^{\lambda_{1}} \cdots\left(\frac{\lambda_{m}}{b_{m}}\right)^{\lambda_{m}} y^{\alpha} \\
= & \lambda_{1} y^{u^{1}}+\cdots+\lambda_{m} y^{u^{m}}-\tilde{c} y^{\alpha} \\
= & \tilde{c}\left(\lambda_{1} y^{u^{1}}+\cdots+\lambda_{m} y^{u^{m}}-y^{\alpha}\right)+(1-\tilde{c})\left(\lambda_{1} y^{u^{1}}+\cdots+\lambda_{m} y^{u^{m}}\right),
\end{aligned}
$$

which is SOS, by Theorem 2.10. This proves (iii). 
Proof of Theorem 2.12. Suppose that such real numbers exist. Condition (i) and Corollary 2.11 imply that $\sum_{i=1}^{m} a_{\alpha, i} x^{u^{i}}+f_{\alpha} x^{\alpha}$ is SOS for every $\alpha \in \Delta$. So

$$
\sum_{\alpha \in \Delta}\left(\sum_{i=1}^{m} a_{\alpha, i} x^{u^{i}}+f_{\alpha} x^{\alpha}\right)
$$

is SOS. By Condition (ii), we have

$$
\sum_{i=1}^{m}\left(f_{u^{i}}-\sum_{\alpha \in \Delta} a_{\alpha, i}\right) x^{u^{i}}
$$

is SOS. Now, it is clear that $f_{\alpha} x^{\alpha}$ is SOS for every $\alpha \notin \Delta$. Therefore,

$$
f(x)=\sum_{i=1}^{m}\left(f_{u^{i}}-\sum_{\alpha \in \Delta} a_{\alpha, i}\right) x^{u^{i}}+\sum_{\alpha \in \Delta}\left(\sum_{i=1}^{m} a_{\alpha, i} x^{u^{i}}+f_{\alpha} x^{\alpha}\right)+\sum_{\alpha \neq \Delta \cup V} f_{\alpha} x^{\alpha}
$$

is $\mathrm{SOS}$.

Proof of Corollary 2.13. Apply Theorem 2.12 with

$$
a_{\alpha, i}=\left|f_{\alpha}\right| \lambda_{\alpha, i} \quad \text { for all } \alpha \in \Delta, i=1, \ldots, m .
$$

Proof of Corollary 2.14. Apply Theorem 2.12 with

$$
a_{\alpha, i}=\left|f_{\alpha}\right| \lambda_{\alpha}^{\lambda_{\alpha}} \quad \text { for all } \alpha \in \Delta, i=1, \ldots, m .
$$

Proof of Corollary 2.15. Apply Theorem 2.12 with

$$
a_{\alpha, i}=\frac{\left|f_{\alpha}\right| \lambda_{\alpha}^{\lambda_{\alpha}} f_{u^{i}}}{\prod_{i=1}^{m} f_{u^{i}}^{\lambda_{i}(\alpha)}} \quad \text { for all } \alpha \in \Delta, i=1, \ldots, m
$$

Proof of Corollary 2.16. Apply Theorem 2.12 with

$$
a_{\alpha, i}= \begin{cases}k \lambda_{\alpha, i}\left(\frac{\left|f_{\alpha}\right|}{k}\right)^{1 / \lambda_{\alpha, i} n_{\alpha}} & \text { if } \lambda_{\alpha, i} \neq 0, \quad \text { for all } \alpha \in \Delta, i=1, \ldots, m . \\ 0 & \text { if } \lambda_{\alpha, i}=0\end{cases}
$$

\section{Application to global optimization}

Let $f \in \mathbf{R}\left[x_{1}, \ldots, x_{n}\right]$ be a real polynomial, and let

$$
f_{*}:=\inf \left\{f(x) \mid x \in \mathbf{R}^{n}\right\} .
$$

Since $f_{*}=\sup \{r \in \mathbf{R} \mid f-r$ is $\operatorname{PSD}\}$, finding $f_{*}$ reduces to determining when $f-r$ is PSD. It is known that deciding when a polynomial is PSD is NP-hard 
[1, Theorem 1.1]. Suppose $\operatorname{deg} f=m$ and $f=f_{0}+\cdots+f_{m}$, where $f_{i}$ is a form of degree $i$. Then a necessary condition for $f_{*} \neq-\infty$ is that $f_{m}$ is PSD, and a sufficient condition for $f_{*} \neq-\infty$ is that $f_{m}$ is PD, i.e., $f_{m}(x)>0$ for all $x \in \mathbf{R}^{n}$ (see [15, Theorem 5.1 and 5.3]). In [9, Theorem 3.1], H. V. Ha and T. S. Pham have given another necessary condition and another sufficient condition for $f_{*} \neq-\infty$ in terms of the Newton polyhedron of $f$, which are improved versions of the results above.

Let

$$
f_{\text {SOS }}:=\sup \{r \in \mathbf{R} \mid f-r \text { is } \operatorname{SOS}\} .
$$

Note that $f_{*} \geq f_{S O S}$. It is known that if $f_{S O S} \neq-\infty$, then $f_{S O S}$ can be computed by semidefinite programming (SDP); see [12, Section 3] and [20]. However, in practice the computation of $f_{S O S}$ can only be carried out if the number of variables and degree are relatively small. The situation is better if $f$ has structured sparsity; see [24, Section 3]. Note that a necessary condition for $f_{S O S} \neq-\infty$ is that $f_{2 d} \in \Sigma_{2 d, n}$ and a sufficient condition for $f_{S O S} \neq-\infty$ is that $f_{2 d} \in \Sigma_{2 d, n}^{0}$, where $\Sigma_{2 d, n}^{0}$ is the interior of $\Sigma_{2 d, n}$ in the vector space of forms of degree $2 d$, equipped with the euclidean topology (see [17, Proposition 5.1]).

Recently, Ghasemi and Marshall have given another lower bounded $f_{g p}$ for the polynomial $f$ of degree $2 d$ with $f_{2 d e_{i}}>0, i=1, \ldots, n$; see [8, Corollary 3.6]. Notice that $f_{g p} \leq f_{S O S}$, and $f_{g p}$ can be computed by geometric programming. As explained in [8, Section 3], although the lower bound found using this method is typically not as good as the lower bound found using SDP, a practical comparision confirms that the computation is faster and larger problems can be handed.

Now, by using Theorem 2.12, we will give a lower bound, which can also be computed by geometric programming, for a larger set of polynomials.

In this section, we also use the term framework to denote a set $\mathscr{U}=$ $\left\{u^{0}, \ldots, u^{m}\right\}$ in which $u^{i}=\left(u_{1}^{i}, \ldots, u_{n}^{i}\right) \in(2 \mathbf{Z})^{n}$ with $u_{j}^{i} \geq 0$, and use the term trellis to denote a framework in which $u^{0}, \ldots, u^{m}$ comprise the vertices of a simplex.

Let $\mathscr{U}$ be a framework. We also use the term $\mathscr{U}$-mediated to denote a set $\mathscr{L} \subset \mathbf{Z}^{n}$ which contains $\mathscr{U}$, and every $v \in \mathscr{L} \backslash \mathscr{U}$ is an average of two distinct even points in $\mathscr{L}$. Also, by [23, Theorem 2.2], it is easly seen that there is a $\mathscr{U}$-mediated set $\mathscr{U}^{*}$ satisfying $\left\{\frac{1}{2}(s+t) \mid s, t \in \mathscr{U}\right\} \subset \mathscr{U}^{*} \subset \operatorname{conv}(\mathscr{U}) \cap \mathbf{Z}^{n}$ which contains every $\mathscr{U}$-mediated set.

Let $V_{\infty}$ denote the set of vertices of $\Gamma_{\infty}(f):=\operatorname{conv}\{\operatorname{supp}(f) \cup 0\}$. We now assume that $V_{\infty}=\left\{u^{0}, \ldots, u^{m}\right\}$ is a trellis with the origin point $u^{0}$. Then for every $\alpha \in \Gamma_{\infty}(f)$, there is only one $\lambda(\alpha)=\left(\lambda_{\alpha, 1}, \ldots, \lambda_{\alpha, m}\right)$ with $\lambda_{\alpha, i} \geq 0$ and $|\lambda(\alpha)|:=\lambda_{1}(\alpha)+\cdots+\lambda_{m}(\alpha) \leq 1$ such that

$$
\alpha=\lambda_{\alpha, 1} u^{1}+\cdots+\lambda_{\alpha, m} u^{m} .
$$

Recall that

$$
\Delta=\left\{\alpha \in \operatorname{supp}(f) \mid \text { either } f_{\alpha}<0 \text { or } \alpha_{i} \text { is odd for some } 1 \leq i \leq n\right\} .
$$


THEOREM 4.1. Let $f$ be a nonconstant polynomial of degree $2 d$, and $r \in \mathbf{R}$. Suppose that $V_{\infty}=\left\{u^{0}, \ldots, u^{m}\right\}$ is a trellis, and $\alpha \in V_{\infty}^{*}$ for all $\alpha \in \Delta$. Then if there exist nonnegative real numbers $a_{\alpha, i}$ for $\alpha \in \Delta, i=1, \ldots, m, a_{\alpha, i}=0$ iff $\lambda_{\alpha, i}=0$, such that

(i) $a_{\alpha}^{\lambda(\alpha)}=\left|f_{\alpha}\right| \lambda(\alpha)^{\lambda(\alpha)}$ for every $\alpha \in \Delta$ such that $|\lambda(\alpha)|=1$,

(ii) $f_{u^{i}} \geq \sum_{\alpha \in \Delta} a_{\alpha, i}, i=1, \ldots, m$,

(iii) $f_{u^{0}}-r \geq \sum_{\alpha \in \Delta^{<1}}(1-|\lambda(\alpha)|)\left[\frac{\left|f_{\alpha}\right| \lambda(\alpha)^{\lambda(\alpha)} \mid}{a_{\alpha}^{\lambda(\alpha)}}\right]^{1 /(1-|\lambda(\alpha)|)}$,

then $f-r$ is SOS. Here $\Delta^{<1}=\{\alpha \in \Delta|| \lambda(\alpha) \mid<1\}$.

Proof. For every $r \in \mathbf{R}$, consider the polynomial $f-r$. By setting $\Omega:=$ $\operatorname{supp}(f) \backslash V_{\infty}$, we can write

$$
f(x)=f_{u^{0}}+\sum_{i=1}^{m} f_{u^{i}} x^{u^{i}}+\sum_{\alpha \in \Omega} f_{\alpha} x^{\alpha} .
$$

There are two cases to consider.

CASE 1: If $r \neq f_{0}$, then the homogenization of $g:=f-r$ is given by

$$
g\left(x_{0}, x\right)=\left(f_{u^{0}}-r\right) x_{0}^{2 d}+\sum_{i=1}^{m} f_{u^{i}} x_{0}^{2 d-\left|u^{i}\right|} x^{u^{i}}+\sum_{\alpha \in \Omega} f_{\alpha} x_{0}^{2 d-|\alpha|} x^{\alpha},
$$

It is not hard to show that the set of vertices of the Newton polyhedron $\Gamma(g)$ of $g$ is the following

$$
\bar{V}_{\infty}=\left\{\bar{u}^{0}=(2 d, 0), \ldots, \bar{u}^{m}=\left(2 d-\left|u^{m}\right|, u^{m}\right)\right\} ;
$$

and moreover,

- $\bar{V}_{\infty}$ is a trellis, since $V_{\infty}$ is a trellis;

- for every $\alpha \in \Delta$, we have $\bar{\alpha}:=(2 d-|\alpha|, \alpha) \in \bar{V}_{\infty}^{*}$, and $\left(1-|\lambda(\alpha)|, \lambda_{\alpha, 1}, \ldots\right.$, $\left.\lambda_{\alpha, m}\right)$ are the barycentric coordinates of $\bar{\alpha}$ with respect to $\bar{V}_{\infty}$;

- by Conditions (i), (ii), and (iii) of Theorem 4.1, there exist nonnegative real numbers $a_{\alpha, i}$ for $\alpha \in \Delta, i=0, \ldots, m$, such that

(i) $a_{\alpha, 0}^{1-|\lambda(\alpha)|} a_{\alpha}^{\lambda(\alpha)}=\left|f_{\alpha}\right|(1-|\lambda(\alpha)|)^{1-|\lambda(\alpha)|} \lambda(\alpha)^{\lambda(\alpha)}$ for $\alpha \in \Delta$,

(ii) $f_{u^{i}} \geq \sum_{\alpha \in \Delta} a_{\alpha, i}, i=1, \ldots, m$, and $f_{u^{0}}-r \geq \sum_{\alpha \in \Delta^{<1}} a_{\alpha, 0}$,

Thus $g$ satisfies the conditions of Theorem 2.12, and so $g$ is SOS. By Proposition 2.1, $f-r$ is SOS.

CASE 2: If $r=f_{0}$, then $\Delta^{<1}=\emptyset$, by Condition (iii) of Theorem 4.1. Then, by Theorem 2.12, Conditions (i) and (ii) of Theorem 4.1 show that $\sum_{i=1}^{m} f_{u^{i}} x^{u^{i}}+$ $\sum_{\alpha \in \Delta} f_{\alpha} x^{\alpha}$ is SOS. Therefore,

$$
f-r=\left(\sum_{i=1}^{m} f_{u^{i}} x^{u^{i}}+\sum_{\alpha \in \Delta} f_{\alpha} x^{\alpha}\right)+\sum_{\alpha \notin \Delta} f_{\alpha} x^{\alpha}
$$

is SOS. 
Definition 4.2. Let $f$ be a nonconstant polynomial of degree $2 d$. Suppose $V_{\infty}=\left\{u^{0}, \ldots, u^{m}\right\}$ is a trellis, and $\alpha \in V_{\infty}^{*}$ for all $\alpha \in \Delta$. We define

$$
\begin{array}{r}
f_{g p}:=\sup \left\{r \in \mathbf{R} \mid \exists a_{\alpha, i} \in \mathbf{R}^{\geq 0}, \alpha \in \Delta, i=1, \ldots, m, a_{\alpha, i}=0 \text { iff } \lambda_{\alpha, i}=0\right. \\
\text { satisfying Conditions (i), (ii), and (iii) of Theorem 4.1 }\} .
\end{array}
$$

Remark 4.3. 1. As a consequence of Theorem 4.1, we have $f_{g p} \leq f_{\text {SOS }}$.

2. If $f_{u^{i}}<0$ for some $i \in\{1, \ldots, m\}$, then $f_{g p}=-\infty$.

COROLlary 4.4. Let $f$ be a nonconstant polynomial of degree $2 d$ such that $V_{\infty}=\left\{u^{0}, \ldots, u^{m}\right\}$ is a trellis. Suppose $\Omega:=\operatorname{supp}(f) \backslash V_{\infty}=\{\alpha\}$, and $\alpha \in V_{\infty}^{*}$ if $\alpha \in \Delta$. Then $f_{*}=f_{\text {SOS }}=f_{g p}$.

Proof. We write

$$
f(x)=f_{u^{0}}+\sum_{i=1}^{m} f_{u^{i}} x^{u^{i}}+f_{\alpha} x^{\alpha} .
$$

Note that $f_{g p} \leq f_{S O S} \leq f_{*}$. So it is sufficient to show that for every real number $r, f_{*} \geq r \Rightarrow f_{g p} \geq r$. Fix $r \in \mathbf{R}$ and assume that $f_{*} \geq r$. We will show $f_{g p} \geq r$; i.e., that $r$ satisfies the constraints of Theorem 4.1. In fact, let $g$ denote the homogenization of $f-r$; i.e.,

$$
g\left(x_{0}, x\right)=\left(f_{u^{0}}-r\right) x_{0}^{2 d}+\sum_{i=1}^{m} f_{u^{i}} x_{0}^{2 d-\left|u^{i}\right|} x^{u^{i}}+f_{\alpha} x_{0}^{2 d-|\alpha|} x^{\alpha} .
$$

Since $f_{*} \geq r$, we get $g \geq 0$.

ClaIM 4.5. We have $f_{u^{i}} \geq 0, i=1, \ldots, m$, and $f_{u^{0}} \geq r$.

Proof. Since $V_{\infty}=\left\{u^{0}, \ldots, u^{m}\right\}$ is a trellis, $\bar{V}_{\infty}=\left\{\bar{u}^{0} \ldots, \bar{u}^{m}\right\}$ is linearly independent in $\mathbf{R}^{n+1}$, where $\bar{u}^{i}=\left(u_{0}^{i}:=2 d-\left|u^{i}\right|, u_{1}^{i}, \ldots, u_{n}^{i}\right), i=0, \ldots, m$. Without the loss of genrerality, we can assume

$$
\operatorname{det}\left[\begin{array}{ccc}
u_{0}^{0} & \cdots & u_{m}^{0} \\
\cdots & \cdots & \cdots \\
u_{0}^{m} & \cdots & u_{m}^{m}
\end{array}\right] \neq 0 .
$$

By the same arguments as in the proof of Corollary 2.11 , for every $\varepsilon \in \mathbf{R}_{>0}$, there exist $c_{0}, \ldots, c_{m} \in \mathbf{R}_{>0}$ such that

$$
\left\{\begin{array}{l}
c_{0}^{u_{0}^{0}} \cdots c_{m}^{u_{m}^{0}}=\varepsilon \\
\cdots \cdots \cdots \cdots \cdots \\
c_{0}^{u_{0}^{m-1}} \cdots c_{m}^{u_{m}^{m-1}}=\varepsilon \\
c_{0}^{u_{0}^{m}} \cdots c_{m}^{u_{m}^{m}}=1 .
\end{array}\right.
$$


Let $\lambda=\left(\lambda_{1}, \ldots, \lambda_{m}\right)$ be such that $\alpha=\lambda_{1} u^{1}+\cdots+\lambda_{m} u^{m}$, with $\lambda_{i} \geq 0$ and $\lambda_{1}+\cdots+\lambda_{m} \leq 1$. Taking $\bar{x}:=\left(c_{0}, \ldots, c_{m}, 1, \ldots, 1\right)$, we have

$$
\begin{aligned}
g(\bar{x})= & \left(f_{u^{0}}-r\right) \bar{x}^{\bar{u}^{0}}+\cdots+f_{u^{m}} \bar{x}^{\bar{u}^{m}}+f_{\alpha}\left(\bar{x}^{\bar{u}^{0}}\right)^{\lambda_{0}} \cdots\left(\bar{x}^{\bar{u}^{m}}\right)^{\lambda_{m}} \quad\left(\lambda_{0}:=1-|\lambda|\right) \\
= & \left(f_{u^{0}}-r\right)\left(c_{0}^{u_{0}^{0}} \cdots c_{m}^{u_{m}^{0}}\right)+\cdots+f_{u^{m}}\left(c_{0}^{u_{0}^{m}} \cdots c_{m}^{u_{m}^{m}}\right) \\
& +f_{\alpha}\left[c_{0}^{u_{0}^{0}} \cdots c_{m}^{u_{m}^{0}}\right]^{\lambda_{0}} \cdots\left[c_{0}^{u_{0}^{m}} \cdots c_{m}^{u_{m}^{m}}\right]^{\lambda_{m}} \\
= & \left(f_{u^{0}}-r+\cdots+f_{u^{m-1}}\right) \varepsilon+f_{u^{m}}+f_{\alpha} \varepsilon^{\lambda_{0}+\cdots+\lambda_{m-1}} .
\end{aligned}
$$

Since $\alpha \in \Omega, \lambda_{0}+\cdots+\lambda_{m-1}>0$. Then, since $g \geq 0$, we have $f_{u^{m}} \geq 0$, by letting $\varepsilon \rightarrow 0$. By the same arguments as the above, we get $f_{u^{i}} \geq 0$ for $i=1, \ldots, m$, and $f_{u^{0}} \geq r$.

To complete the proof of Corollary 4.4, let us consider two cases.

Case 1: Assume $f_{\alpha}>0$ and all $\alpha_{i}$ are even. Then $\alpha \notin \Delta$, and so $\Delta=\emptyset$. In this case, $r$ satisfies trivially the constraints of Theorem 4.1. So $f_{g p} \geq r$.

CASE 2: Assume either $f_{\alpha}<0$ or not all $\alpha_{i}$ are even. Then $\alpha \in \Delta$; i.e., $\Delta=\Omega=\{\alpha\}$. In this case, applying Corollary 2.11 to $g$, we deduce that

$$
\left|f_{\alpha}\right| \lambda^{\lambda}(1-|\lambda|)^{1-|\lambda|} \leq \prod_{i=1}^{m} f_{u^{i}}^{\lambda_{i}}\left(f_{u^{0}}-r\right)^{1-|\lambda|} .
$$

There are two subcases to consider.

- If $|\lambda|<1$, then $r$ satisfies the constraints of Theorem 4.1 with

$$
a_{\alpha, i}= \begin{cases}f_{u^{i}} & \text { if } \lambda_{i} \neq 0, \\ 0 & \text { if } \lambda_{i}=0 .\end{cases}
$$

- If $|\lambda(\alpha)|=1$, then $\left|f_{\alpha}\right| \lambda^{\lambda} \leq \prod_{i=1}^{m} f_{u^{i}}^{\lambda_{i}}$, and so $r$ satisfies the constraints of Theorem 4.1 with

$$
a_{\alpha, i}= \begin{cases}s f_{u^{i}} & \text { if } \lambda_{i} \neq 0 \\ 0 & \text { if } \lambda_{i}=0\end{cases}
$$

where

$$
s=\frac{\left|f_{\alpha}\right| \lambda^{\lambda}}{\prod_{i=1}^{m} f_{u^{i}}^{\lambda_{i}}}
$$

Therefore, $f_{g p} \geq r$. form

Definition 4.6 (geometric program). (1) A function $\phi: \mathbf{R}_{>0}^{n} \rightarrow \mathbf{R}$ of the

$$
\phi(x)=c x_{1}^{a_{1}} \cdots x_{n}^{a_{n}}
$$


where $c>0, a_{i} \in \mathbf{R}$, and $x=\left(x_{1}, \ldots, x_{n}\right)$, is called a monomial function. A sum of monomial functions, i.e., a function of the form

$$
\phi(x)=\sum_{i=1}^{k} c_{i} x_{1}^{a_{1 i}} \cdots x_{n}^{a_{n i}},
$$

where $c_{i}>0$ for $i=1, \ldots, k$, is called a posynomial function.

(2) An optimization problem of form

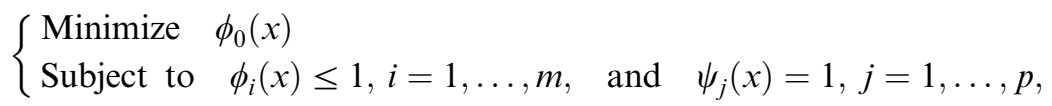

where $\phi_{0}, \ldots, \phi_{m}$ are posynomials and $\psi_{0}, \ldots, \psi_{p}$ are monomial functions, is called a geometric program (GP). The subset of $\mathbf{R}_{>0}^{n}$ defined by the constrants

$$
\phi_{i}(x) \leq 1, \quad i=1, \ldots, m, \quad \text { and } \quad \psi_{j}(x)=1, \quad j=1, \ldots, p,
$$

is called the feasible set of the GP. $\phi_{0}(x)$ is called the objective function. The output of the GP is the minimum, more precisely, the infimum, of $\phi_{0}(x)$, taken as $x$ runs through the feasible set. In case feasible set is empty, the output is understood to be $+\infty$.

See [3, Section 4.5] or [21, Section 3.5] for more about GPs.

THEOREM 4.7. Let $f$ be a nonconstant polynomial of degree $2 d$. Suppose that

(i) $V_{\infty}=\left\{u^{0}, \ldots, u^{m}\right\}$ is a trellis,

(ii) $\alpha \in V_{\infty}^{*}$ for all $\alpha \in \Delta$.

Then $f_{g p}=f_{0}-r^{*}$, where $r^{*}$ is the output of the GP

$$
\left\{\begin{array}{l}
\text { Minimize } \sum_{\alpha \in \Delta^{<1}}(1-|\lambda(\alpha)|)\left[\frac{\left|f_{\alpha}\right| \lambda(\alpha)^{\lambda(\alpha)} \mid}{a_{\alpha}^{\lambda(\alpha)}}\right]^{1 /(1-|\lambda(\alpha)|)} \\
\text { Subject to } \sum_{\alpha \in \Delta} \frac{a_{\alpha, i}}{f_{u^{i}}} \leq 1, i=1, \ldots, m, \quad \text { and } \\
\frac{a_{\alpha}^{\lambda(\alpha)}}{\left|f_{\alpha}\right| \lambda(\alpha)^{\lambda(\alpha)}}=1, \alpha \in \Delta,|\lambda(\alpha)|=1 .
\end{array}\right.
$$

The variables in the program are the $a_{\alpha, i}, \alpha \in \Delta, i=1, \ldots, m, \lambda_{i}(\alpha) \neq 0$, the understanding being that $a_{\alpha, i}=0$ iff $\lambda_{i}(\alpha)=0$.

Proof. Observe that

$$
\phi_{0}(a):=\sum_{\alpha \in \Delta^{<1}}(1-|\lambda(\alpha)|)\left[\frac{\left|f_{\alpha}\right| \lambda(\alpha)^{\lambda(\alpha)} \mid}{a_{\alpha}^{\lambda(\alpha)}}\right]^{1 /(1-|\lambda(\alpha)|)}
$$


and

$$
\phi_{i}(a):=\sum_{\alpha \in \Delta} \frac{a_{\alpha, i}}{f_{u^{i}}}, \quad i=1, \ldots, m,
$$

are posynomials in the variables $a_{\alpha, i}$, and

$$
\psi_{\alpha}(a):=\frac{a_{\alpha}^{\lambda(\alpha)}}{\left|f_{\alpha}\right| \lambda(\alpha)^{\lambda(\alpha)}}, \quad \alpha \in \Delta,|\lambda(\alpha)|=1,
$$

are monomial functions in the variables $a_{\alpha, i}$. By the definition of $f_{g p}$, it follows immediately that $f_{g p}=f_{0}-r^{*}$. that

COROLlary 4.8. Let $f$ be a nonconstant polynomial of degree $2 d$. Suppose

(i) $V_{\infty}=\left\{u^{0}, \ldots, u^{m}\right\}$ is a trellis,

(ii) $\alpha \in V_{\infty}^{*}$ for all $\alpha \in \Delta$.

Then if $|\lambda(\alpha)|<1$ for each $\alpha \in \Delta$, then $f_{g p} \neq-\infty$ and $f_{g p}=f_{0}-r^{*}$, where $r^{*}$ is the output of the GP

$$
\left\{\begin{array}{l}
\text { Minimize } \sum_{\alpha \in \Delta}(1-|\lambda(\alpha)|)\left[\frac{\left|f_{\alpha}\right| \lambda(\alpha)^{\lambda(\alpha)} \mid}{a_{\alpha}^{\lambda(\alpha)}}\right]^{1 / 1-|\lambda(\alpha)|} \\
\text { Subject to } \quad \sum_{\alpha \in \Delta} \frac{a_{\alpha, i}}{f_{u^{i}}} \leq 1, i=1, \ldots, m
\end{array}\right.
$$

Proof. In this case, the equality constraints in the computation of $r^{*}$ are vacuous and the feasible set is always nonempty, so $f_{g p} \neq-\infty$. The rest is immediate from Theorem 4.7.

We establish some lower bounds in terms of its coefficients, which can be obtained by evaluating the objective function of the GP in Corollary 4.8 at suitably chosen feasible points. Corollaries 4.9, 4.10, and 4.11 are improved versions of [8, Corollaries 4.1, 4.2, and 4.3] (and so [7, Theorems 3.1, 3.2, and 3.3]) respectively.

Recall that for a (univariate) polynomial of the form $p(t)=t^{n}-\sum_{i=0}^{n-1} a_{i} t^{i}$, where each $a_{i}$ is nonnegative and at least one $a_{i}$ is nonzero, there is a unique positive root of $p$ (see [19, Theorem 1.1.3]). Let $C(p)$ denote the unique positive root of $p$. See [5], [11, Example 4.6.2], and [8, Proposition 1.2] for more details and upper bounds for $C(p)$. that

COROLlary 4.9. Let $f$ be a nonconstant polynomial of degree $2 d$. Suppose

(i) $V=\left\{u^{0}, \ldots, u^{m}\right\}$ is a trellis,

(ii) $\alpha \in V^{*}$ for all $\alpha \in \Delta$, and

(iii) $|\lambda(\alpha)|<1$ for each $\alpha \in \Delta$. 
Then $f_{g p} \geq r_{L}$, where

$$
\begin{aligned}
& r_{L}:=f_{0}-\sum_{\alpha \in \Delta}(1-|\lambda(\alpha)|)\left|f_{\alpha}\right| k^{2 D|\lambda(\alpha)|} f_{V}^{-\lambda(\alpha)} \\
& k \geq \max _{i=1, \ldots, m} C\left(t^{2 D}-\sum_{\alpha \in \Delta} \lambda_{\alpha, i}\left|f_{\alpha}\right| f_{u^{i}}^{-|\lambda(\alpha)|} t^{2 D|\lambda(\alpha)|}\right) .
\end{aligned}
$$

Here $f_{V}:=\left(f_{u^{1}}, \ldots, f_{u^{m}}\right)$, and $D$ is a nonnegative integer number such that $2 D \lambda_{\alpha, i} \in \mathbf{Z}$ for all $\alpha \in \Delta, i=1, \ldots, m$.

Proof. For each $\alpha \in \Delta$ and $i=1, \ldots, m$, let

$$
a_{\alpha, i}=\frac{\lambda_{\alpha, i}\left|f_{\alpha}\right| f_{u^{i}}^{1-|\lambda(\alpha)|}}{k^{2 D(1-\lambda(\alpha))}} .
$$

By the defintion of $k$, for each $i=1, \ldots, m$, we have $\sum_{\alpha \in \Delta} \lambda_{\alpha, i}\left|f_{\alpha}\right| f_{u^{i}}^{-|\lambda(\alpha)|} k^{2 D|\lambda(\alpha)|}$ $\leq k^{2 D}$. Hence

$$
\sum_{\alpha \in \Delta} a_{\alpha, i}=\sum_{\alpha \in \Delta} \frac{\lambda_{\alpha, i}\left|f_{\alpha}\right| f_{u^{i}}^{1-|\lambda(\alpha)|}}{k^{2 D(1-\lambda(\alpha))}} \leq f_{u^{i}}
$$

This shows that the array $\left(a_{\alpha, i} \mid \alpha \in \Delta, i=1, \ldots, m\right)$ is a feasible point for the geometric program in the statement of Corrolary 4.8. Plugging this into the objective function of the program yields

$$
\begin{aligned}
\sum_{\alpha \in \Delta} & (1-|\lambda(\alpha)|)\left[\frac{\left|f_{\alpha}\right| \lambda(\alpha)^{\lambda(\alpha)} \mid}{a_{\alpha}^{\lambda(\alpha)}}\right]^{1 /(1-|\lambda(\alpha)|)} \\
& =\sum_{\alpha \in \Delta}(1-|\lambda(\alpha)|)\left[\left|f_{\alpha}\right| \prod_{\lambda_{\alpha, i} \neq 0}\left(\frac{\lambda_{\alpha, i}}{a_{\alpha, i}}\right)^{\lambda_{\alpha, i}}\right]^{1 /(1-|\lambda(\alpha)|)} \\
& =\sum_{\alpha \in \Delta}(1-|\lambda(\alpha)|)\left[\left|f_{\alpha}\right| \prod_{\lambda_{\alpha, i} \neq 0}\left(\frac{\lambda_{\alpha, i} k^{2 D(1-\lambda(\alpha))}}{\lambda_{\alpha, i}\left|f_{\alpha}\right| f_{u^{i}}^{1-|\lambda(\alpha)|}}\right)^{\lambda_{\alpha, i}}\right]^{1 /(1-|\lambda(\alpha)|)} \\
& =\sum_{\alpha \in \Delta}(1-|\lambda(\alpha)|)\left|f_{\alpha}\right| k^{2 D|\lambda(\alpha)|} \prod_{\lambda_{i}(\alpha) \neq 0} f_{u^{i}}^{-\lambda_{i}(\alpha)} \\
& =\sum_{\alpha \in \Delta}(1-|\lambda(\alpha)|)\left|f_{\alpha}\right| k^{2 D|\lambda(\alpha)|} f_{V}^{-\lambda(\alpha)}
\end{aligned}
$$

So, $r_{L}=f_{0}-\sum_{\alpha \in \Delta}(1-|\lambda(\alpha)|)\left|f_{\alpha}\right| k^{2 D|\lambda(\alpha)|} f_{V}^{-\lambda(\alpha)} \leq f_{g p}$. 
COROLlary 4.10. Let $f$ be a nonconstant polynomial of degree $2 d$. Suppose that

(i) $V=\left\{u^{0}, \ldots, u^{m}\right\}$ is a trellis,

(ii) $\alpha \in V^{*}$ for all $\alpha \in \Delta$, and

(iii) $|\lambda(\alpha)|<1$ for each $\alpha \in \Delta$.

Then $f_{g p} \geq r_{F K}$, where $r_{F K}:=f_{0}-k^{2 D}$, with

$$
\begin{aligned}
& k \geq C\left(t^{2 D}-\sum_{j=1}^{2 D-1} b_{j} t^{j}\right), \quad \text { and } \\
& b_{j}:=\left(\frac{2 D-j}{2 D}\right)^{(2 D-j) / 2 D} \sum_{\alpha \in \Delta, 2 D|\lambda(\alpha)|=j}\left|f_{\alpha}\right| \lambda(\alpha)^{\lambda(\alpha)} f_{V}^{-\lambda(\alpha)}, \quad j=1, \ldots, 2 D-1 .
\end{aligned}
$$

Here $D$ is a nonnegative integer number such that $2 D \lambda_{i}(\alpha) \in \mathbf{Z}$ for all $\alpha \in \Delta$, $i=1, \ldots, m$.

Proof. For each $\alpha \in \Delta$ and $i=1, \ldots, m$, let

$$
a_{\alpha, i}=(1-|\lambda(\alpha)|)^{1-|\lambda(\alpha)|}\left|f_{\alpha}\right| \lambda(\alpha)^{\lambda(\alpha)} f_{V}^{-\lambda(\alpha)} f_{u^{i}} k^{j-2 D} .
$$

By the defintion of $k$, we have $\sum_{j=1}^{2 D-1} b_{j} k^{j} \leq k^{2 D}$. Hence

$$
\begin{aligned}
\sum_{\alpha \in \Delta} a_{\alpha, i} & =\sum_{\alpha \in \Delta}(1-|\lambda(\alpha)|)^{1-|\lambda(\alpha)|}\left|f_{\alpha}\right| \lambda(\alpha)^{\lambda(\alpha)} f_{V}^{-\lambda(\alpha)} f_{u^{i}} k^{j-2 D} \\
& =\sum_{j=1}^{2 D-1} \sum_{\alpha \in \Delta, 2 D|\lambda(\alpha)|=j}\left(\frac{2 D-j}{2 D}\right)^{(2 D-j) / 2 D}\left|f_{\alpha}\right| \lambda(\alpha)^{\lambda(\alpha)} f_{V}^{-\lambda(\alpha)} f_{u^{i}} k^{j-2 D} \\
& =f_{u^{i}} \sum_{j=1}^{2 D-1} k^{j-2 D}\left(\frac{2 D-j}{2 D}\right)^{(2 D-j) / 2 D} \sum_{\alpha \in \Delta, 2 D|\lambda(\alpha)|=j}\left|f_{\alpha}\right| \lambda(\alpha)^{\lambda(\alpha)} f_{V}^{-\lambda(\alpha)} \\
& =f_{u^{i}} k^{-2 D} \sum_{j=1}^{2 D-1} k^{j}\left(\frac{2 D-j}{2 D}\right)^{(2 D-j) / 2 D} \sum_{\alpha \in \Delta, 2 D|\lambda(\alpha)|=j}\left|f_{\alpha}\right| \lambda(\alpha)^{\lambda(\alpha)} f_{V}^{-\lambda(\alpha)} \\
& =f_{u^{i}} k^{-2 D} \sum_{j=1}^{2 D-1} b_{j} k^{j} \leq f_{u^{i}} .
\end{aligned}
$$

This shows that the array $\left(a_{\alpha, i} \mid \alpha \in \Delta, i=1, \ldots, m\right)$ is a feasible point for the geometric program in the statement of Corrolary 4.8. Plugging this into the objective function of the program yields 


$$
\begin{aligned}
& \sum_{\alpha \in \Delta}(1-|\lambda(\alpha)|)\left[\frac{\left|f_{\alpha}\right| \lambda(\alpha)^{\lambda(\alpha)} \mid}{a_{\alpha}^{\lambda(\alpha)}}\right]^{1 /(1-|\lambda(\alpha)|)} \\
& =\sum_{\alpha \in \Delta}(1-|\lambda(\alpha)|)\left[\left|f_{\alpha}\right| \prod_{\lambda_{\alpha, i} \neq 0}\left(\frac{\lambda_{\alpha, i}}{a_{\alpha, i}}\right)^{\lambda_{\alpha, i}}\right]^{1 /(1-|\lambda(\alpha)|)} \\
& =\sum_{\alpha \in \Delta}(1-|\lambda(\alpha)|)\left[\left|f_{\alpha}\right| \prod_{\lambda_{\alpha, i} \neq 0}\left(\frac{f_{V}^{\lambda(\alpha)} k^{2 D-j} \lambda_{\alpha, i}}{(1-|\lambda(\alpha)|)^{1-|\lambda(\alpha)|}\left|f_{\alpha}\right| \lambda(\alpha)^{\lambda(\alpha)} f_{u^{i}}}\right)^{\lambda_{\alpha, i}}\right]^{1 /(1-|\lambda(\alpha)|)} \\
& =\sum_{j=1}^{2 D-1} \sum_{\alpha \in \Delta, 2 D|\lambda(\alpha)|=j}\left(\frac{2 D-j}{2 D}\right) \\
& \times\left[\left|f_{\alpha}\right| \prod_{\lambda_{\alpha, i} \neq 0}\left(\frac{f_{V}^{\lambda(\alpha)} k^{2 D-j} \lambda_{\alpha, i}}{\left(\frac{2 D-j}{2 D}\right)^{(2 D-j) / 2 D}\left|f_{\alpha}\right| \lambda(\alpha)^{\lambda(\alpha)} f_{u^{i}}}\right]^{\lambda_{\alpha, i}}\right]^{2 D /(2 D-j)} \\
& =\sum_{j=1}^{2 D-1} k^{j}\left(\frac{2 D-j}{2 D}\right)^{(2 D-j) / 2 D} \sum_{\alpha \in \Delta, 2 D|\lambda(\alpha)|=j}\left|f_{\alpha}\right| \lambda(\alpha)^{\lambda(\alpha)} f_{V}^{-\lambda(\alpha)} \\
& =\sum_{j=1}^{2 D-1} b_{j} k^{j} \leq k^{2 D} \text {. }
\end{aligned}
$$

So, $r_{F K}=f_{0}-k^{2 D} \leq f_{g p}$.

COROLlary 4.11. Let $f$ be a nonconstant polynomial of degree $2 d$. Suppose that

(i) $V=\left\{u^{0}, \ldots, u^{m}\right\}$ is a trellis,

(ii) $\alpha \in V^{*}$ for all $\alpha \in \Delta$, and

Then

(iii) $|\lambda(\alpha)|<1$ for each $\alpha \in \Delta$.

$$
f_{g p} \geq r_{r m t}:=f_{0}-\sum_{\alpha \in \Delta}(1-|\lambda(\alpha)|)\left[\left|f_{\alpha}\right| t^{|\lambda(\alpha)|} \lambda(\alpha)^{\lambda(\alpha)} f_{V}^{-\lambda(\alpha)}\right]^{1 /(1-|\lambda(\alpha)|)},
$$

where $t:=|\Delta|$.

Proof. For each $\alpha \in \Delta$ and $i=1, \ldots, m$, let $a_{\alpha, i}=\frac{f_{u^{i}}}{t}$. Then

$$
\sum_{\alpha \in \Delta} a_{\alpha, i}=\sum_{\alpha \in \Delta} \frac{f_{u^{i}}}{t}=f_{u^{i}} .
$$


This shows that the array $\left(a_{\alpha, i} \mid \alpha \in \Delta, i=1, \ldots, m\right)$ is a feasible point for the geometric program in the statement of Corrolary 4.8. Plugging this into the objective function of the program yields

$$
\begin{aligned}
\sum_{\alpha \in \Delta} & (1-|\lambda(\alpha)|)\left[\frac{\left|f_{\alpha}\right| \lambda(\alpha)^{\lambda(\alpha)} \mid}{a_{\alpha}^{\lambda(\alpha)}}\right]^{1 /(1-|\lambda(\alpha)|)} \\
= & \sum_{\alpha \in \Delta}(1-|\lambda(\alpha)|)\left[\left|f_{\alpha}\right| \prod_{\lambda_{\alpha, i} \neq 0}\left(\frac{\lambda_{\alpha, i}}{a_{\alpha, i}}\right)^{\lambda_{\alpha, i}}\right]^{1 /(1-|\lambda(\alpha)|)} \\
= & \sum_{\alpha \in \Delta}(1-|\lambda(\alpha)|)\left[\left|f_{\alpha}\right| \prod_{\lambda_{\alpha, i} \neq 0}\left(\frac{t \lambda_{\alpha, i}}{f_{u^{i}}}\right)^{\lambda_{\alpha, i}}\right]^{1 /(1-|\lambda(\alpha)|)} \\
= & \sum_{\alpha \in \Delta}(1-|\lambda(\alpha)|)\left[\left|f_{\alpha}\right| t^{|\lambda(\alpha)|} \lambda(\alpha)^{\lambda(\alpha)} f_{V}^{-\lambda(\alpha)}\right]^{1 /(1-|\lambda(\alpha)|)} .
\end{aligned}
$$

So, $r_{d m t}=f_{0}-\sum_{\alpha \in \Delta}(1-|\lambda(\alpha)|)\left[\left|f_{\alpha}\right| t^{|\lambda(\alpha)|} \lambda(\alpha)^{\lambda(\alpha)} f_{V}^{-\lambda(\alpha)}\right]^{1 /(1-|\lambda(\alpha)|)} \leq f_{g p}$.

Acknowledgments. The authors are greatly indebted to Ha Huy Vui for suggesting the problem and for many stimulating conversations.

\section{REFERENCES}

[1] M. Bellare AND P. Rogaway, The complexity of approximating a nonlinear program, Math. Program. 69 (1993), 429-441.

[2] J. Bochnak, M. Coste And M. F. Roy, Real algebraic geometry, Ergeb. Math. Grenzgeb. 36, Springer, New York, 1998.

[ 3 ] S. Boyd and L. Vandenberghe, Convex optimization, Cambridge University Press, Cambridge, 2004.

[ 4 ] M. D. Choi, T. Y. Lam And B. Reznick, Sum of squares of real polynomials, Proc. Sympos. Pure Math. 58 (1995), 103-126.

[ 5 ] E. Deutsch, Bounds for the zeros of polynomial, Amer. Math. Monthly 88 (1981), 205-206.

[6] C. Fidalgo AND A. Kovacec, Positive semidefinite diagonal minus tail forms are sum of squares, Math. Z. 269 (2011), 629-645.

[ 7 ] M. Ghasemi AND M. Marshall, Lower bounds for polynomials in terms of its coefficients, Arch. Math. 95 (2010), 343-354.

[ 8 ] M. Ghasemi AND M. Marshall, Lower bounds for polynomials using geometric programming, SIAM J. Optim. 22 (2012), 460-473.

[9] H. V. HA AND T. S. Pham, Minimizing polynomial functions, Acta Math. Vietnamica 32 (2007), 71-82.

[10] D. Hilbert, Uber die Darstellung definiter Formen als Summe von Formenquadraten, Math. Ann. 32 (1888), 342-350 (in German).

[11] D. KNUTH, The art of computer programming 2, Addison-Wesley, New York, 1969.

[12] J. B. LASSERRE, Global optimization with polynomials and the problem of moments, SIAM J. Optim. 11 (2001), 796-817. 
[13] J. B. Lasserre, Sufficient conditions for a real polynomial to be a sum of squares, Arch. Math. (Basel) 89 (2007), 390-398.

[14] J. B. LASSERRE AND T. NETZER, SOS approximation of nonnegative polynomial via simple high degree perturbations, Math. Z. 256 (2006), 99-112.

[15] M. Marshall, Optimization of polynomials functions, Canad. Math. Bull. 46 (2008), 537-587.

[16] M. Marshall, Positive polynomials and sum of squares, Math. Serveys Monogr. 146, American Mathematical Society, Providence, RI, 2008.

[17] M. Marshall, Representation of non-negative polynomials, degree bounds and application to optimization, Canad. J. Math. 61 (2009), 205-221.

[18] T. Motzkin, The arithmetic-geometric inequality, Inequalities (O. Shisha, ed.), Academic Press, New York, 1970, 205-224.

[19] V. V. Prasolov, Polynomials, Algorithms comput. math. 11, Springer, New York, 2004.

[20] P. Parrilo and B. Sturmeels, Minimizing polynomial functions, DiMACS Serie in Discrite Mathematics and Theoretical Computer Science 60, AMS, 2003, 83-99.

[21] A. L. Peressini, F. E. Sullivan and J. J. Uhl, JR, The mathematics of nonlinear programming, Undergrad. texts math., Springer, New York, 1987.

[22] B. ReZnick, Midpoint polytopes and the map $x_{i} \rightarrow x_{i}^{k}$, in preparation.

[23] B. Reznick, Forms derived from the arithmetic-geometric inequality, Math. Ann. 383 (1989), 431-464.

[24] H. Waki, S. Kim, M. Kojima And M. Muramatsu, Sum of squarer and semidefinite program relaxations for polynomials optimization problems with structured sparsity, SIAM J. Optim. 17 (2006), 218-242.

\author{
Van Doat Dang \\ Thang Long High School for GIFTed \\ 10 Tran Phu, Da Lat \\ VIETNAM \\ E-mail: dangvandoat@yahoo.com.vn \\ Thi Thao Nguyen \\ DePARTMENT OF MATHEMATICS \\ Hanoi National University of Education \\ 136 Xuan Thuy Road \\ Cau Giay District, Hanoi \\ VIETNAM \\ E-mail: math_thao@yahoo.com.vn
}

